\title{
Desarrollo en las infancias institucionalizadas y en familias de acogida temporal: Una revisión bibliográfica
}

\author{
Development in institutionalized infants and temporary shelter families:
}

\author{
A bibliographic review
}

\author{
María Paula Moretti ${ }^{1}$ y Norma Mariana Torrecilla ${ }^{2}$
}

\begin{abstract}
${ }^{1}$ Licenciada en Psicología. Docente de la Facultad de Humanidades y Ciencias Económicas, Universidad Católica Argentina, Mendoza. Miembro del Equipo Técnico del Programa de Familia Temporaria de la Asociación de Voluntarios de Mendoza (AVOME), Mendoza, Argentina.

E-mail: morettimariapaula@gmail.com.
\end{abstract}

${ }^{2}$ Doctora en Psicología. Investigadora Asistente del Consejo Nacional de Investigaciones Científicas y Técnicas (CONICET) en el Instituto de Medicina y Biología Experimental de Cuyo Centro Científico Tecnológico (IMBECU - CCT), Mendoza, Argentina. Docente de la Facultad de Humanidades y Ciencias Económicas, Universidad Católica Argentina, Mendoza.

E-mail: maritorrecilla@yahoo.com.ar.

\section{Resumen}

El objetivo del presente trabajo fue realizar una revisión bibliográfica sobre el desarrollo de los niños y niñas bajo cuidados alternativos institucionales y de familias de acogida temporal. Se realizó en diversas bases de datos científicas: Science Direct, SciELO, Redalyc, PubMed, DialNet y Google Académico, utilizando las siguientes palabras claves: "desarrollo infantil", "familias de acogida temporal", "institucionalización", "child development", "temporary family foster care", "residential care". Fueron seleccionados 58 artículos, publicados en revistas científicas de los últimos 17 años (período 2000-2017). Se incluye una síntesis de sus resultados organizando a la misma en dos apartados: en el primero se hace referencia a las condiciones de vida en el ámbito institucional y al desarrollo infantil en dichas condiciones, $\mathrm{y}$ en el segundo, a los programas de familia de acogida temporal y al desarrollo tras la inclusión en estos programas. Se destacan los aspectos que restan profundizar.

Palabras clave: Desarrollo Infantil; Familias de acogida temporal; Institucionalización.

\begin{abstract}
In different parts of the world, family is considered the main responsible for ensuring children the full enjoyment of their rights; also, family is recognized as the central core for children's development. Even so, in exceptional situations, children need to be separated from their biological family. In those cases, alternative care is required. This study investigates two different types of alternative care: institutional care and family foster care. The aim of this paper is to review the existing literature on child development of children in those types of alternative care. The
\end{abstract}


review was done through different scientific data base: Science Direct, SciELO, Redalyc, PubMed, DialNet y Academic Google, and using the following key words: "desarrollo infantile", "familias de acogida temporal", "institucionalización", "child development", "temporary family foster care", "residential care". With reference to the studies about temporary family foster care, the ones considered were those that made reference to family systems that took temporary care of a child until their judicial situation was solved. These were external families that have no bonds with the child. Those studies in which the families were pre-adoptive ones were not considered. Fifty-eight articles, published in the last seventeen years (from 2000 to 2017), were chosen. From this total, nineteen correspond to systematic revisions, four are reports from children organizations, while the remaining thirty-five correspond to empiric studies. The results of this paper are organized in two different sections: the first one makes reference to the conditions of life at an institution and the child development under these conditions, whereas the second one makes reference to temporary family foster care programs and the child development after the inclusion in these programs. As regards the first section, it is concluded that the institutional care shows the lack of an exclusive and stable affect figure, being the children exposed to unstable rotating caregivers with consistent bond disruption. These caregivers are described as unavailable to the children with minimal emotional and physical contact. Furthermore, the caregivers show lack of empathy and little responsiveness to child's individual needs resulting in chronic emotional deprivation. At the same time, the above mentioned is closely related to the delays or difficulties that the development of these institutionalized children show in different areas: cognitive, intellectual, academic, socio-affective, emotional, physical-motor, cerebral and neurological. In the second section of the paper, the results of the studies on children development in tempo- rary family foster care are described and the countries where evidence on these programs was found are presented. They show that there are significant and beneficial physical, emotional, socio-affective, behavioral, cognitive and intellectual changes in the development of institutionalized children after they enter temporary family foster care. Moreover, the development of children living in alternative care differs significantly from the one of those living with family foster care. The results also specify the characteristics that a temporary family foster care must have in order to facilitate the child's development. It is concluded that children in institutional care show delays and maladaptation in various domains of development. Besides, the longer the child is institutionalized, the more his behavior is affected. The authors believe that these institutions are not the right places to raise children during a long period of time and they propose the need to prioritize temporary family foster care. The results underline the importance of family foster care programs as an alternative that could improve children's development. Nevertheless, institutional care is still the predominant alternative worldwide for children without parental care. Finally, this systematic review aims to be a starting point for future research. The aspects that need to be deepen are highlighted.

Keywords: Child development; Temporary family foster care; Residential care.

\section{Introducción}

Largo fue el camino sociocultural y jurídico transitado por la infancia para arribar a su concepción actual bajo el paradigma de protección integral. En gran parte de la historia, los niños se encontraron desfavorecidos en su trato, siendo sometidos a dinámicas de maltrato social y familiar, manteniéndose cosificados, sin voz participativa en la sociedad y como objetos de represión.

Fue recién en el año 1989 cuando se 
comenzaron a sentar las bases jurídicas necesarias para un viraje en esta historia. En dicho año, la Asamblea General de las Naciones Unidas adoptó la Convención Internacional de los Derechos del Niño (CDN). Fue el primer instrumento jurídico que reconoce a los niños y niñas como sujetos sociales y de derechos, generando una revolución y cambio de paradigma respecto de la infancia. Ello conllevó a cambios legislativos en distintos países. Específicamente en Argentina, la Ley de Protección Integral de Derechos de Niñas, Niños y Adolescentes (NNA) (Ley 26.061), sancionada en el año 2005, garantizó la puesta en marcha de los principios contemplados en la CDN, adecuando la legislación interna a los postulados de esta última. Es a partir de ello que el paradigma de protección integral comienza a instaurarse generando nuevas demandas y desafíos (UNICEF, 2012).

En diversas partes del mundo, se reconoce a la familia como la principal responsable de asegurar a NNA el pleno disfrute y ejercicio de sus derechos, y como núcleo central de protección de la infancia (Palummo, 2013). El derecho a la vida en familia está consagrado en la CDN (1989), en la cual, desde su preámbulo se reconoce "que el niño, para el pleno y armonioso desarrollo de su personalidad, debe crecer en el seno de la familia, en un ambiente de felicidad, amor y comprensión". Específicamente en Argentina, la Ley $\mathrm{N}^{\circ} 26.061$ (2005), identifica a la familia como la principal responsable del cuidado del NNA (Art. $7^{\circ}$ ) y plantea, como derecho de todo NNA, el desarrollo en su ámbito familiar de origen (Art. $\left.11^{\circ}\right)$. Sin embargo, en casos excepcionales, y por distintos motivos, pueden ser separados de sus progenitores y familias de origen. En estos casos, se requiere de cuidados alternativos brindados desde el Estado y la sociedad, siendo los cuidados institucionales (hogares) una posibilidad en este cuidado alternativo. Si bien los espacios institucionales son una posibilidad de cuidado, son reconocidos como posibles causas de perjuicios a los NNA debiendo ser limitados a tiempos breves
(Palummo, 2013). Todo lo descripto conllevó a impulsar movimientos de desinstitucionalización, priorizando el cuidado familiar por sobre los institucionales. Dichos movimientos han sido, por un lado, en relación a la prevención o asistencia a la familia de origen, como así también, por otro lado, a la generación y fortalecimiento de programas de acogimiento familiar temporario, como otro cuidado alternativo transitorio posible, para permitir protección del NNA desde un ámbito familiar hasta la resolución de su situación judicial (Ley 26.061, 2005; Palummo, 2013; UNICEF, 2012, 2014).

Es de fundamental importancia destacar leyes provinciales posteriores a la $\mathrm{CDN}$, que propusieron también estrategias concretas de desinstitucionalización. En la provincia de Mendoza, en 1995 se sancionó y promulgó la Ley de Protección Integral del niño y del adolescente (Ley $\mathrm{N}^{\circ}$ 6.354). La misma tiene por objeto la protección integral del niño y del adolescente, como sujeto de derechos, en el núcleo familiar (Art. $1^{\circ}$ y Art. $2^{\circ}$ ). Por otro lado, en Ciudad Autónoma de Buenos Aires (CABA) se promulgó en el 2007, la Ley de Acogimiento Familiar (Ley $\mathrm{N}^{\circ}$ 2.213). En ella se especifican objetivos, beneficiarios, función, requisitos y exclusiones, responsabilidades, límites, duración, revocación y autoridad de aplicación del acogimiento familiar en Buenos Aires. Asimismo, define a este último como: "el cuidado de forma integral, temporal y no institucional, brindado por una familia alternativa de convivencia a un niño, niña o adolescente, cuando medie inexistencia de su grupo familiar de pertenencia, se encuentre privado de él en forma temporal, o exista medida judicial administrativa, en razón de causas o motivos suficientes para ordenar la separación de su medio familiar" (Art. $2^{\circ}$ ).

A partir de lo descripto, el objetivo del presente trabajo de investigación fue realizar una revisión bibliográfica sobre el desarrollo de aquellos niños y niñas que no pueden convivir con su familia biológica por vulneración de derechos, manteniéndose, por lo tanto, 
bajo cuidados alternativos institucionalizados, o bien, en familias de acogida temporal, hasta la resolución de su situación legal. Se busca también conocer lo especificado en la bibliografía sobre los cambios en el desarrollo de estos niños y niñas previamente institucionalizados tras su ingreso a una familia de acogida temporal.

\section{Método}

El estudio consistió en una revisión de la literatura en diversas bases de datos científicas:

SCIENCE DIRECT, SCIELO, REDALYC, PUBMED, DIALNET y GOOGLE ACADÉMICO, utilizando las siguientes palabras clave: desarrollo infantil, familias de acogida temporal, institucionalización, child development, temporary family foster care, residential care.

Se consideraron los artículos publicados en revistas científicas de los últimos 17 años (período 2000-2017), pertenecientes a investigaciones realizadas en diversos países del mundo. En cuanto a los estudios sobre familias de acogida temporal (temporary family foster care) se incorporaron sólo aquellos que hacían referencia a sistemas familiares que temporalmente cuidaban a uno o más niños o niñas hasta la resolución de su situación judicial, sin mantener vínculo de parentesco con el infante (familias ajenas). No se consideraron aquellos estudios en donde esas familias eran preadoptivas (tendientes a convertirse en adoptivas), ni estudios con muestras constituidas sólo por familias de acogida cuyos miembros sostenían algún vínculo de parentesco con el niño o niña.

A partir de lo descripto, fueron seleccionados 58 artículos, de los cuales 19 corresponden a revisiones sistemáticas (Anghel, Herczog, y Dima, 2013; Bakermans-Kranenburg et al., 2011; Browne, 2017; Del Valle, Bravo y López López, 2009; Finet et al., 2016; García Quiroga y Hamilton-Giachritsis, 2016; Gómez Bengoechea y Berástegui Pedro Viejo, 2009; Gunnar y Quevedo, 2007; Heim y Nemeroff, 2001; Jaar y Córdova, 2017; Johnson y Gunnar, 2011; Kaufman et al., 2000; Martínez González, 2008; McCall, 2012; Monaghan y Haussmann, 2015; Nemeroff, 2004; Rodríguez Juárez, 2016; Van IJzen- doorn et al., 2011; World Health Organization, 2004), 4 son informes de organizaciones para la infancia (Palummo, 2013; RELAF, 2016; UNICEF, 2012; 2014), mientras que los 35 restantes corresponden a estudios empíricos.

En cuanto a las características metodológicas de estos estudios empíricos, sería importante destacar los siguientes datos, sintetizados en la Figura 1:

- 27 presentan metodología cuantitativa, 20 de ellos con diseño transversal, de los cuales uno tiene un alcance descriptivo (López López, et al., 2010), y los 19 restantes un alcance correccional (Almas et al., 2014; Chugani et al., 2001; Eluvathingal et al., 2006; García-Baamonde Sánchez, 2008; Govindan et al., 2010; Groark, et al., 2011; Herce et al., 2003; Kertes et al., 2008; Levin et al., 2015; Martín, et al., 2008; Martín, García y Siverio, 2012; Martín, Muñoz de Bustillo Díaz y Pérez, 2011; Mehta et al., 2009; Montserrat Boada, Casas Aznar y Bertran, 2013; Moreno Manso, García-Baamonde Sánchez y Blázquez Alonso, 2010; Moreno Manso, Rabazzo Méndez y García-Baamonde, 2006; Moulson et al., 2014; Palacios, Moreno y Román, 2013; Roy y Rutter, 2005). Los otros siete estudios cuantitativos son longitudinales, con alcance correlacional. De ellos, cinco son no experimentales (Beckett et al., 2002; Fernández Millán, Hamido-Mohamed y Ortiz Gómez, 2009; Fox et al., 2011; Kang et al., 2014; O'Connor, et al., 2000) y dos con diseño experimental (McLaughlin et al., 2011; Nelson et al., 2007)

- Sólo uno de los artículos empíricos seleccionados presenta una metodología cualitativa, con alcance descriptivo (Del Valle, 
López López, Montserrat y Bravo, 2008).

-Siete presentan una metodología mixta: cuatro de ellos con diseño transversal y alcance correlacional (Dumitrescu, 2016; López López et al., 2011; Smyke et al.,
2007; Valgañón, 2014), y los otros tres, con diseño longitudinal, descriptivos-correlacionales (Amorós et al., 2003; Molero Mañes, Gil Llario, y Díaz Rodríguez, 2014; Molero Mañes y Moral Valderas, 2009).

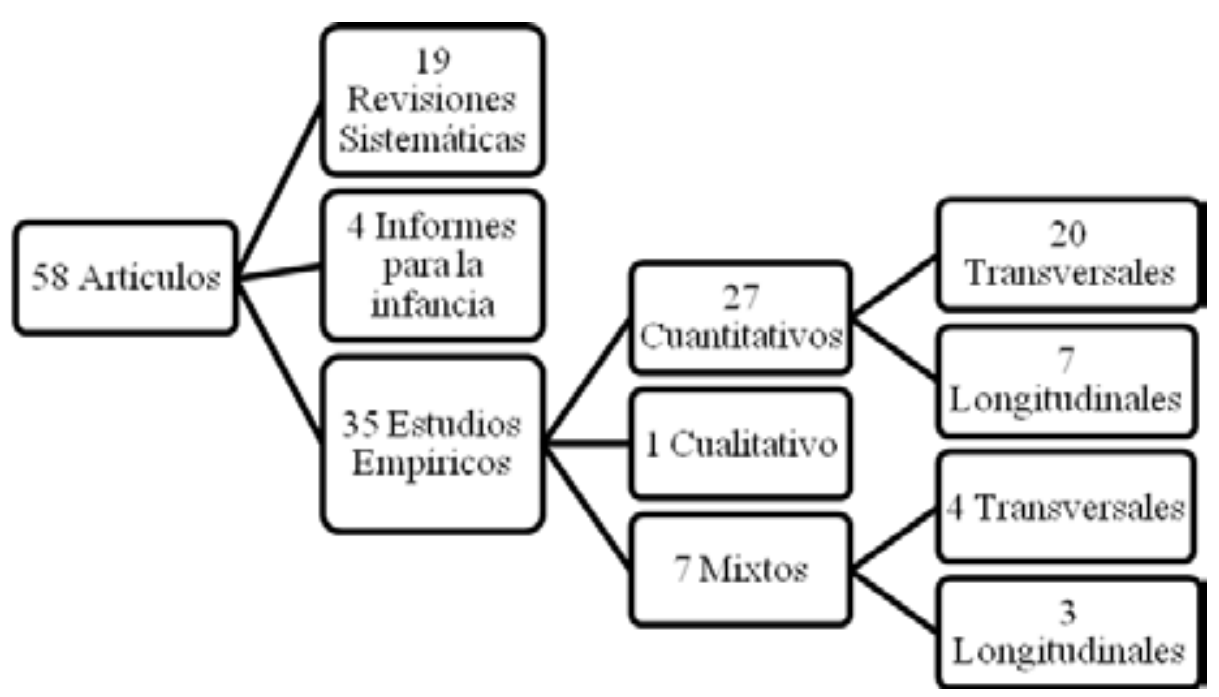

Figura 1. Clasificación metodológica de los artículos seleccionados.

Con respecto a las muestras de estos 35 estudios empíricos, podría realizarse la siguiente clasificación:

- Un total de diez de estos estudios empíricos presentan una muestra constituida por niños/as institucionalizados (en hogares). Tres de ellos estudian también a niños/as no institucionalizados (Martín et al., 2008; Martín, García y Siverio, 2012; Martín, Muñoz de Bustillo Díaz y Pérez, 2011), mientras que los otros siete sólo estudian a los niños/as de estas instituciones (Fernández Millán, Hamido-Mohamed y Ortiz Gómez, 2009; García-Baamonde Sánchez, 2008; Groark et al., 2011; López López et al., 2011; Moreno Manso, García-Baamonde Sánchez y Blázquez Alonso, 2010; Moreno Manso, Rabazzo Méndez y García-Baamonde, 2006; Smyke et al., 2007). Uno de ellos describe también las condiciones de las instituciones y aspectos vinculares (Groark et al., 2011).

- Ocho estudian niños/as o adolescentes adoptados con historia en instituciones. Cinco de ellos estudian también a un grupo de niños/as o adolescentes sin historia de institucionalización (Chugani et al., 2001; Eluvathingal et al., 2006; Govindan et al., 2010; Mehta et al., 2009; Roy y Rutter, 2005). Los otros tres, sólo estudian niños adoptados con historia de institucionalización (Beckett et al., 2002; Kertes et al., 2008; O'Connor et al., 2000).

- Siete de ellas estudian dos grupos de niños/ as que se encontraban, al momento de la evaluación, en cuidados alternativos, uno en instituciones (hogares) y otro en familias de acogida. Cinco de ellos comparan sus resultados con niños/as sin cuidados alternativos (Fox et al., 2011; McLaughlin et al., 2011; Moulson et al., 2014; Nelson et al., 2007; Palacios, Moreno y Román, 2013), mientras que los otros dos sólo trabajan con los dos grupos mencionados, de niños en cuidados alternativos (Kang et al., 2014; Montserrat Boada, Casas Aznar y Bertran, 2013).

- Cinco de estas investigaciones empíricas estudian niños/as en familias de acogida, formando parte estas familias de la muestra 
(Amorós et al., 2003; Dumitrescu, 2016; Herce et al., 2003; López López, et al., 2010; Valgañón, 2014).

- Otras dos investigaciones estudian específicamente a las familias de acogida temporal (Molero Mañes, Gil Llario y Díaz Rodríguez, 2014; Molero Mañes y Moral Valderas, 2009).

- Dos investigaciones estudian dos grupos de niños/as con antecedentes en cuidados alternativos, uno con historia en instituciones y otro con historia en familias de acogida. Uno de ellos administra instrumentos de evalua- ción a estos dos grupos de niños (Almas et al., 2014), mientras que otro busca describir y relacionar variables de ambos grupos, a partir de cuestionarios administrados a sus padres o cuidadores (Levin et al., 2015).

- Uno de estos estudios empíricos presenta muestras constituidas sólo por niños/as con historia (antecedentes) en familias de acogida (Del Valle et al., 2008)

En la Figura 2 se presenta una síntesis de las descripciones muestrales de los 35 estudios empíricos que conforman parte de la presente revisión.

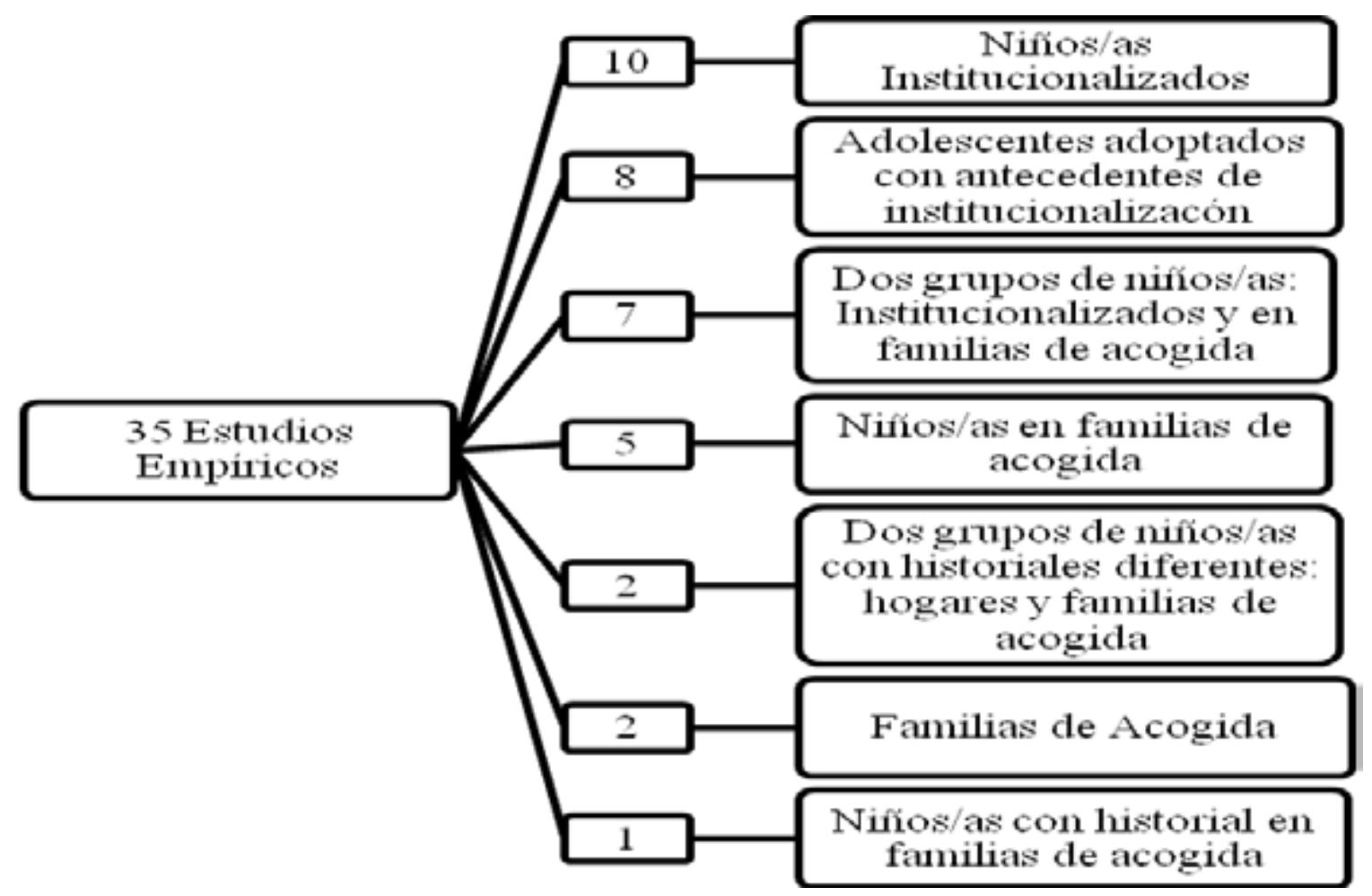

Figura 2. Descripciones muestrales de estudios empíricos.

\section{Resultados}

\section{Condiciones del cuidado institucional}

En los artículos seleccionados pueden identificarse diversas condiciones de vida institucional y tipos de cuidados con los que crecen los niños/as institucionalizados.

Spitz (1945, citado en Gómez Bengoechea y Berástegui Pedro Viejo, 2009) espe- cifica que suelen estar dados los elementos físicos para satisfacer aquellas necesidades básicas como la alimentación, higiene y seguridad física. En nuestro país, un relevamiento realizado por UNICEF (2012) observa condiciones materiales y edilicias predominantemente buenas logrando superar los estándares mínimos exigidos para la atención de los niños. Otros autores, han concluido que se trata de ambientes con cierta escasez de 
recursos físicos, falta de espacios necesarios (espacios de privacidad o privados para que los niños, niñas y adolescentes guarden sus pertenencias, ausencia de espacios de recreación, etc.) y de materiales adecuados para brindar seguridad y protección (presencia de extintores, certificados de salubridad, salidas de emergencias, entre otros) (Palummo, 2013; RELAF, 2016; Rodríguez Juárez, 2016; Van IJzendoorn et al., 2011).

En relación a ello, informes sobre situación actual de NNA de América Latina y del Caribe bajo cuidados institucionales, especifican que las instituciones deberían estar situadas en lugares céntricos. Esto implica que presenten acceso a diversos servicios como medios de transportes, educación, salud, ocio, entre otros, que posibiliten integración social y comunitaria. Sin embargo, identifican una gran cantidad de instituciones que no logran cumplir con ello, encontrándose alejadas de zonas céntricas y urbanas, generando desentrenamiento social y falta de contacto con el mundo exterior (Palummo, 2013; RELAF, 2016).

Por otro lado, la mayoría de las investigaciones concuerdan en la presencia de diversas fallas a nivel del personal responsable para el despliegue de su rol de cuidado (Bakermans Kranenburg et al., 2011; Groark et al., 2011; Jaar y Córdova, 2017; Martínez González, 2008; Palummo, 2013; Rodríguez Juárez, 2016; Spitz, 1945, citado en Gómez Bengoechea y Berástegui Pedro Viejo, 2009; Van IJzendoorn et al., 2011).

Especifican que los niños y niñas institucionalizados carecen de una figura de afecto exclusiva y estable, siendo expuestos a cuidadores rotativos con inestabilidad y disrupciones constantes de vínculos. (Bakermans Kranenburg et al., 2011; Jaar y Córdova, 2017; Martínez González, 2008; Palummo, 2013; RELAF, 2016; Van IJzendoorn et al., 2011). Ya Spitz (1945, citado en Gómez Bengoechea y Berástegui Pedro Viejo, 2009) observaba dicha rotación y describía la escasa cantidad de cuidadores por niño. Esto último se relaciona con la dificultad para lograr una atención personalizada e incapacidad para percibir necesidades específicas de cada niño (Palummo, 2013), generando efecto de masificación (RELAF, 2016; Rodríguez Juárez, 2016).

A su vez, refieren que estos adultos no se encuentran previamente preparados o con formación suficiente para ejercer el delicado rol de cuidado (Jaar y Córdova, 2017; Van IJzendoorn et al., 2011) y que, en su gran mayoría, presentan pocos niveles de motivación y predisposición para la tarea (Jaar y Córdova, 2017). Palummo (2013) especifica que en Latinoamérica y Caribe, el personal no siempre es contratado teniendo en cuenta experiencia y capacitación con niños. Describe que ello se debe, en gran parte, a falta de condiciones laborales, baja remuneración y a la ausencia de criterios para contratación.

Por otro lado, se sostiene que los cuidadores se muestran poco accesibles y disponibles para los niños, manteniendo escaso contacto físico e interacciones socioemocionales. Se han revelado niveles pobres de sensibilidad (habilidad para leer e interpretar señales), calidez, empatía y capacidad de respuesta de los cuidadores ante las necesidades de los niños. Ellos quedan expuestos a vínculos afectivos con muchas carencias, lo que se ha llamado carencia afectiva crónica en los vínculos (Groark et al., 2011; Jaar y Córdova, 2017; Martínez González, 2008; Smyke et al., 2007; Spitz, 1945, citado en Gómez Bengoechea y Berástegui Pedro Viejo, 2009; Van IJzendoorn et al., 2011).

El informe sobre cuidados institucionales en América Latina y Caribe (Palummo, 2013), describe la variable del traslado, indicando que los niños suelen residir en distintas instituciones a lo largo de su estadía en cuidados alternativos, lo cual implicaría mayor dificultad para el establecimiento de un vínculo emocional estable con cuidadores y otros niños. De la mano de ello, especifica que, si bien algunos países (como Brasil, Chile, Colombia y México) intentan mantener el 
vínculo entre hermanos, la mayoría de los países latinoamericanos utilizan el criterio de clasificación por edad, conllevando en varias oportunidades a la falta de protección de vínculos fraternales.

Jaar y Córdova (2017) y el informe realizado por RELAF (2016) describen a los tiempos de institucionalización como prolongados, con demoras excesivas, manteniendo a lactantes, niños y niñas en eternas esperas bajo las condiciones previamente mencionadas. Estas estadías prolongadas representan un problema de extrema gravedad. Un relevamiento realizado en instituciones de la Ciudad de Buenos Aires, Argentina, indica que un 40 $\%$ de los NNA tienen una estadía promedio de entre 3 y 6 años (RELAF, 2016). Se propone revisar esta cuestión de las demoras en las medidas excepcionales considerando que los tiempos manejados por los adultos e instituciones no son los tiempos necesarios para el bienestar de un niño, vulnerando nuevamente sus derechos (Palummo, 2013; RELAF, 2016; UNICEF, 2012).

\section{Desarrollo de niños y niñas institucionalizados}

Diversos estudios indican la existencia de retrasos y dificultades en el desarrollo y salud psicofísica del niño institucionalizado, estableciendo relación entre dichos retrasos con los tipos de cuidado y vínculos propios de vida institucional previamente descriptos (Almas et al., 2014; Bakermans-Kranenburg et al., 2011; Beckett et al., 2002; Bowlby, 1969, citado en World Health Organization, 2004; Bowlby, 1979, citado en Rodríguez Juárez, 2016; Chugani et al., 2001; Eluvathingal et al., 2006; Fernández Millan, Hamido-Mohamed y Ortiz Gómez, 2009; Finet et al., 2016; Fox et al., 2011; García-Baamonde Sánchez, 2008; Govindan et al., 2010; Groark et al., 2011; Kertes et al., 2008; López López et al., 2011; McCall, 2012; Martín et al., 2008: Martín, García y Siverio, 2012; Martín, Muñoz de Bustillo y Pérez, 2011; Mehta et al., 2009; Moreno Manso; García-Baamonde Sánchez y Blázquez Alonso, 2010; Moreno Manso, Rabazo Méndez y García-Baamonde, 2006; Moulson et al., 2014; Palummo, 2013; RELAF, 2016; Smyke et al., 2007; Roy y Rutter, 2005; Spitz, 1945, citado en Gómez Bengoechea y Berástegui Pedro Viejo, 2009; Van IJzendoorn et al., 2011).

\section{Desarrollo Cognitivo.}

En cuanto al retraso en el desarrollo infantil, algunas investigaciones se centran en el aspecto cognitivo, arribando a la presencia de un cociente intelectual $(\mathrm{CI})$ y rendimiento cognitivo general relativamente bajo en niños con historia de institucionalización (Finet et al., 2016; Fox et al., 2011; Smyke et al., 2007; Van IJzendoorn et al., 2011). McCall (2012) revela deficiencias en las funciones ejecutivas de atención, memoria y planificación. En relación a ello, se ha constatado dificultad para mantener atención necesaria para la resolución de problemas (Moreno Manso, Rabazo Méndez y García Baamonde, 2006) y dificultades en la capacidad de abstracción (Bowlby, 1979, citado en Rodríguez Juárez, 2016).

Desde un enfoque más específico, varios estudios demuestran existencia de retrasos en el desarrollo cognitivo lingüístico en niños y niñas institucionalizados o con historia de institucionalización, presentando un nivel por debajo de la edad cronológica, con dificultades en el dominio de la morfología, sintáctica, pragmática y semántica (Bowlby, 1979, citado en Rodríguez Juárez, 2016; García-Baamonde Sánchez, 2008; Moreno Manso, García-Baamonde Sánchez y Blázquez Alonso, 2010; Moreno Manso, Rabazo Méndez y García-Baamonde, 2006; Van IJzendoorn et al., 2011). Se especifican retrasos o dificultades para: hacer uso de pronombres, formas verbales irregulares pasadas y futuras y de la voz pasiva; ponerse en el lugar del interlocutor adulto; utilizar al lenguaje como medio para solicitar atención de manera satisfactoria y como medio para 
autorregular el comportamiento; mantener una continuidad en el discurso sin saltar de un tema al otro; solicitar más información o aclaraciones; y expresar desagrado y disgusto de forma adecuada (Moreno Manso, García-Baamonde Sánchez y Blázquez Alonso, 2010). Almas et al. (2014) revelan mayores niveles de inhibición en el uso del lenguaje en niños y niñas institucionalizados, mostrándose más reticentes para expresarse verbalmente. A su vez, en este mismo grupo de infantes se han identificado retrasos en el aprendizaje de la lectura (Roy y Rutter, 2005).

En relación a esto último planteado por Roy y Rutter (2005), sería importante agregar que existen diversos estudios argentinos sobre el desarrollo de la capacidad de lectoescritura de todo niño, niña y adolescente, en los que se destaca la importancia y necesidad de un contexto y entorno social familiar favorable para un desarrollo adaptativo de dicha capacidad. Cartocetiet al. (2016) destacan grandes dificultades en la comprensión de textos en niños, niñas y adolescentes con entornos sociales y educativos desfavorables. También Márquez, Iparraguirre y Bengtsson (2015) especifican que padres $u$ otros adultos de cuidado del niño ejercen un rol clave para su desarrollo en la escritura. Describen que dicho rol se debe dar a partir de una participación activa y presencial en prácticas de lectura y escritura, y especifican que los contextos sociofamiliares desfavorecidos se relacionan con dificultades en el desarrollo de esta capacidad de escritura en el niño.

En dirección al área académica y escolar, algunos autores postulan que los niños institucionalizados presentan inadaptación a dicho ámbito, siendo esta manifestada mediante hipolaboriosidad, requerimiento de apoyo para alcanzar logros académicos, desagrado y baja motivación por el estudio y aprendizaje, indisciplina y problemas de conducta en el aula, aversión al docente e insatisfacción escolar (García-Baamonde Sánchez, 2008; López López et al., 2011; Martín et al., 2008; Moreno Manso, García Baamonde Sánchez y
Blázquez Alonso, 2010).

\section{Desarrollo socioafectivo.}

Otra área indagada con respecto al desarrollo del niño institucionalizado ha sido la socioafectiva y emocional. Con respecto a esta variable, los resultados de numerosas investigaciones revelan que el tipo de apego predominantemente manifestado en estos niños es el inseguro y desorganizado (García Quiroga y Hamilton-Giachritsis, 2016; Van IJzendoorn et al., 2011). Referido a ello, otros autores describen la expresión de un comportamiento amistoso, desinhibido e indiscriminado, ante cualquier adulto, incluso ante extraños, en ausencia de sensaciones de miedo o precaución ante lo desconocido (Bakermans-Kranenburg et al., 2011; Groark et al., 2011; McCall, 2012; RELAF, 2016). Además, los comportamientos disruptivos de carácter agresivo han sido observados $\mathrm{y}$ revelados como altamente presentes en el vínculo con el otro (Fernández Millan, Hamido-Mohamed y Ortiz Gómez, 2009; Groark et al., 2011), como así también, altos niveles de ansiedad (García-Baamonde Sánchez, 2008) y pobres habilidades sociales (Palacios, Moreno y Román, 2013), dificultando la integración con los demás (Fernández Millán, Hamido-Mohamed y Ortiz Gómez, 2008; García-Baamonde Sánchez, 2008; Groark et al., 2011; Palacios, Moreno y Román, 2013). Asimismo, se ha observado a largo plazo, cierto deterioro en la capacidad para establecer y mantener lazos afectivos significativos y duraderos, lo cual ha sido denominado carácter insensible (Bowlby, 1979, citado en Rodríguez Juárez, 2016).

En relación a estas fallas en la integración con los demás se podría retomar el ámbito escolar a nivel vincular y socio relacional con sus pares. Los resultados encontrados indican grandes dificultades a la hora de establecer relaciones de amistad en el ámbito de las tareas académicas, en donde los niños institucionalizados son significativamente menos 
elegidos y más rechazados por los demás compañeros, hecho que no se observa en el ámbito de actividades de ocio, juego y recreativas. Estos resultados localizan los problemas de relación interpersonal con los iguales en los contextos formales, dentro del aula, lo que dificulta el logro de una buena adaptación y del éxito escolar (Martín et al., 2008; Martín, Muñoz de Bustillo y Pérez, 2011). Un estudio más reciente realizado por Martín, García y Siverio (2012) concluye que los niños en acogimiento residencial obtienen mayores puntuaciones en escalas de inadaptación autopercibida al contexto escolar que niños no institucionalizados.

Otro aspecto estudiado en relación al desarrollo emocional ha sido la capacidad para el reconocimiento de las emociones del otro a partir de la lectura de sus expresiones faciales y la capacidad para guiar las decisiones sociales tras dicha lectura. Un estudio indica que los niños en cuidados alternativos institucionales presentan mayor dificultad en la capacidad del reconocimiento, sobre todo en las emociones de felicidad, tristeza, miedo y enojo. A su vez, se observan ciertas fallas en la capacidad de responder de manera acertada a esas expresiones (Moulson et al., 2014).

Considerando aspectos psicopatológicos, se sostiene que la crianza temprana en instituciones se encuentra asociada con un aumento del riesgo en la conexión y comunicación social y trastornos del espectro autista (Levin et al., 2015). Spitz (1945, citado en Gómez Bengoechea y Berástegui Pedro Viejo, 2009) describe estados depresivos bajo los cuales suelen encontrarse estos niños, llevando a desórdenes alimentarios (como descenso en la ingesta de alimentos) y cierta inmunodepresión, conduciendo a un deterioro físico progresivo, aumento de prevalencia de infecciones y elevada tasa de mortalidad. Beckett et al. (2002) revelan problemas emocionales y conductuales expresados mediante el cuerpo en niños adoptados con historia de institucionalización, tales como: balanceo, autolesiones y desórdenes alimentarios, con dificultades en la masticación y deglución de alimentos sólidos. Esto se relaciona con teorías psicológicas que identifican a los primeros objetos de amor del niño (figuras principales de cuidado) como dadores de personalidad, identidad e imagen corporal. Son aquellas figuras principales de cuidado las que serán internalizadas por el niño, a modo especular e inconsciente. Estas huellas pueden darse en falta, generando la sintomatología y patologías descriptas anteriormente (Dolto, 1979, citado en Velázquez, 2014).

El informe de RELAF (2016) describe el fenómeno de despersonalización, indicando dificultad en estos NNA que crecen en contexto institucional para considerarse a sí mismos, sin tener presentes sus deseos, sentimientos y aspiraciones personales.

\section{Desarrollo físico y neurológico y sus efectos en otras áreas del desarrollo.}

En lo referente al desarrollo físico, se han identificado retrasos madurativos en el crecimiento, relacionados con un bajo peso y menor estatura y tamaño de la cabeza del niño con historia de institucionalización (López López, 2011; RELAF, 2016; Smyke et al., 2007; Van IJzendoorn et al., 2011), con dificultades para el logro de una coordinación motora acorde a la edad cronológica (Spitz, 1945 citado en Gómez Bengoechea y Berástegui Pedro Viejo, 2009).

En cuanto al desarrollo y funcionamiento neurológico, se concluye que se ve afectado de manera negativa por la exposición a experiencias tempranas adversas y a factores estresantes durante la infancia temprana (incluso a lo largo de la vida prenatal intrauterina). Una variable extensamente estudiada ha sido la regulación del eje hipotalámico-hipofisario-adrenal (eje HHA) y la producción de la hormona cortisol, revelando daños en la regulación de dicho eje y producción atípica de esta hormona debido a la exposición temprana a estresores. Esa afectación puede conllevar un aumento significativo del riesgo de mani- 
festar posteriormente problemas físicos y trastornos mentales, entre los que se han mencionado la depresión, trastornos de ansiedad $\mathrm{u}$ otros trastornos psiquiátricos y una mayor vulnerabilidad, tanto en la infancia como en la adultez, ante factores estresores (dificultad en la capacidad de respuesta ante el estresor) (Gunnar y Quevedo, 2007; Heim and Nemeroff, 2001; Kaufman et al., 2000; Monaghan y Haussmann, 2015; Nemeroff, 2004). Algunas investigaciones descubren este proceso en niños institucionalizados o con historia de institucionalización, sosteniendo a la crianza en instituciones como el factor estresante que genera los daños y riesgos anteriormente descriptos (Kertes et al., 2008; Van IJzendoorn et al., 2011).

Otros estudios se han centrado en el desarrollo neurológico de niños y adolescentes adoptados que presentan historia de institucionalización en su temprana infancia, utilizando como grupo control niños $y$ adolescentes sin experiencias de institucionalización. Mencionan disfunciones en regiones cerebrales prefrontales, frontales, temporales y parietales en niños con historia de institucionalización. Entre ellas se revelan: disminución de materia gris y blanca, anomalías en sistema límbico, volumen engrandecido de amígdala particularmente en el hemisferio derecho, falla en conexiones entre dichas áreas. Especifican que se trata de áreas vinculadas a las funciones ejecutivas y emoción, generando déficits y retrasos en desarrollo cognitivo, socioemocional y comportamental, denotando falta de atención, hiperactividad, altos niveles de impulsividad con fallas en el control inhibitorio y regulación emocional (Chugani et al., 2001; Eluvathingal et al., 2006; Govindan et al., 2010; Mehta et al., 2009).

\section{Relación con otras variables.}

McCall (2012) aclara que estos retrasos o dificultades en el desarrollo no pueden ser atribuidos específicamente a la crianza institucional, indicando que, sin lugar a dudas, la genética, condiciones prenatales (como las exposiciones a factores teratógenos), complicaciones durante el nacimiento y experiencias familiares previas a la institucionalización (vivencias de maltrato infantil) son factores influyentes. Sin embargo, concuerda con que la crianza en una institución contribuye, y en gran medida, a las mismas. Asimismo, Van IJzendoorn et al. (2011) concluyen que no todo niño es afectado en la misma manera y proporción ante el cuidado alternativo institucional, sino que ello dependerá de su temperamento y características de personalidad específicas. En consonancia con ello, algunos investigadores han desarrollado y discutido el término de resiliencia. Indican que la exposición temprana a leves niveles de estrés puede ser beneficiosa para algunos lactantes y niños ya que les puede posibilitar el desarrollo de su capacidad de resiliencia ante el estrés para momentos posteriores de la vida. Sin embargo, especifican que dependerá de los niveles del estrés temprano y de la personalidad del sujeto (Monaghan y Haussmann, 2015; Van IJzendoorn et al., 2011).

Otra variable estudiada ha sido el tiempo de institucionalización transcurrido. Diversos autores concluyen que los niños con mayor estancia institucional son los más vulnerables siendo que en ellos se observa una alta incidencia de afectación en las distintas áreas de su desarrollo y salud, conllevando a una mayor dificultad para alcanzar parámetros de desarrollo esperables para su edad cronológica, incluso tras finalizar su vida institucional y convivir con una familia (ya sea biológica, adoptiva o temporal) (Beckett et al., 2002; Fernández Millan, Hamido-Mohamed y Ortiz Gómez, 2009; Govindan et al., 2010; Johnson y Gunnar, 2014; López López et al., 2011; McCall,2012; Mehta et al., 2009; O'Connor et al., 2000).

Por último, en cuanto al período de institucionalización, se ha identificado a los primeros dos años de vida como aquellos más sensibles, por lo que la exposición a la crianza en una institución durante este tiempo puede generar 
detenimiento y retrasos graves en el desarrollo del lactante, pudiendo ser de largo plazo y hasta incluso permanentes, sin lograr cambios tras la finalización de la vida institucional y su posterior ingreso a una familia (McCall, 2012; Palummo, 2013; Van IJzendoorn et al., 2011; Spitz, 1945, citado en Gómez Bengoechea y Berástegui Pedro Viejo, 2009).

A modo de conclusión, en cuanto a las condiciones y el desarrollo psicofísico de los niños institucionalizados, los autores sostienen que en ningún caso los hogares de niños deben ser lugares para crecer y desarrollarse durante largos intervalos de tiempo, remarcando que deben ser considerados sólo como espacios de emergencia y como último recurso. A su vez, postulan la necesidad de priorizar la alternativa del acogimiento familiar temporal (Browne, 2017; Jaar y Córdova, 2017; López López et al., 2011; Martínez González, 2008; Palummo, 2013).

\section{Programas de familias de acogida}

La revisión de la literatura permite revelar existencia de Programas de Familia de Acogida Temporal en diversos países del mundo, describiéndola como una alternativa de cuidado del niño que no puede, por el momento, permanecer con sus progenitores y familia de origen y tampoco convivir con familia adoptiva. Más específicamente se observó en países europeos tales como España (Amorós et al., 2003; Del Valle, Bravo y López López, 2009; Del Valle et al., 2008; Herce et al., 2003; López López et al., 2010; Martínez González, 2008; Molero Mañes, Gil Llario y Díaz Rodríguez, 2014; Molero Mañes y Moral Valderas, 2009; Montserrat Boada, Casas Aznar y Bertrán, 2013), Rumania (Almas et al., 2014; Anghel, Herczog y Dima, 2013; Dumitrescu, 2016; Fox et al., 2011; Levin et al., 2015; McLaughlin et al., 2011; Nelson et al., 2007), Hungría (Anghel, Herczog y Dima, 2013), países asiáticos como Corea (Kang et al., 2014) y países latinoamericanos y del Caribe como Chile (Jaar y Córdova, 2017),
Argentina (Valgañón, 2014), Paraguay, El Salvador, Honduras, Guatemala, República Dominicana, Perú, Brasil (Palummo, 2013).

Asimismo, a partir de la revisión de la literatura se podrían construir tres fundamentos diferentes para la creación y existencia de estos programas. En primera instancia, se deben a los principios planteados por la CDN y a las diversas legislaciones de cada nación desde el paradigma de protección de la infancia. Se posiciona al niño como sujeto de derecho, protegiendo su derecho a desarrollarse en un ambiente familiar, comprendiendo a la unidad familiar como la principal responsable de su contención (UNICEF, 2012). El segundo fundamento es aquel anteriormente descripto por investigadores sobre el desarrollo del niño en el ámbito institucional, es decir, para evitar las consecuencias negativas de la institucionalización en el desarrollo y crecimiento de estos niños. Se trata de un fundamento con fines predominantemente preventivos (Browne, 2017; Jaar y Córdova, 2017; Martínez González, 2008; López López et al., 2011). Un tercer fundamento observado ha sido aquel brindado por estudios comparativos entre niños institucionalizados y niños en programas de acogimiento familiar temporario, o bien, entre un mismo grupo de niños institucionalizados tras su ingreso a dichos programas (Almas et al., 2014; Amorós et al., 2003; Del Valle, López López, Montserrat y Bravo, 2008; Fox et al., 2011; Kang et al., 2014; Levin et al., 2015; McLaughlin et al., 2011; Montserrat Boada, Casas Aznar y Bertrán, 2013; Nelson et al., 2007). A continuación, se presentan las conclusiones y resultados de estos últimos estudios comparativos.

Dos de estos estudios comparaban el desarrollo de un grupo de niños institucionalizados antes y luego de su ingreso a programas de familias temporarias. Permiten concluir que existen cambios cuantitativos y cualitativos significativos en cuanto al desarrollo del niño institucionalizado tras el ingreso a una familia de acogida temporal (Amorós et al., 2003; Del Valle et al., 2008). Vale mencionar que la 
posibilidad de cambios estará relacionada al tiempo y período transcurrido en la institucionalización (McCall, 2012), tal como se especificó en el apartado anterior.

Más específicamente, a nivel físico, los valores de peso y estatura alcanzan los valores esperables para la edad cronológica tras el ingreso a una familia temporaria, disminuyendo problemáticas de salud física o de crecimiento. También se observan mejoras en el área intelectual y del lenguaje tras el ingreso del niño, repercutiendo de manera positiva en el rendimiento escolar y procesos de adquisición de aprendizaje. En cuanto al comportamiento, los autores han encontrado mejoras en la higiene, alimentación y hábitos de autonomía con mayor predisposición a ser ayudado (Amorós et al., 2003; Del Valle et al., 2008). Por último, Amorós et al. (2003) también destacan cambios con respecto al desarrollo emocional y socioafectivo, observando apegos más seguros, mejoras en la capacidad de reconocimiento y expresión de emociones, mayores niveles de autoconfianza y un aumento significativo en la habilidad de integración con el entorno y pares.

Por otro lado, diversos fueron los estudios encontrados sobre comparaciones entre distintos grupos de niños, comparando el desarrollo de niños institucionalizados del de niños previamente institucionalizados que fueron incluidos en programas de familias de acogida temporal. Vale aclarar que uno de estos estudios fue realizado con adolescentes. Permiten arribar a conclusiones similares a las anteriores, observando un desarrollo psicofísico más positivo en niños incluidos en programas de familias temporarias que en el de niños que permanecen institucionalizados (Almas et al., 2014; Fox et al., 2011; Kang et al., 2014; Levin et al., 2015; McLaughlin et al., 2011; Montserrat Boada, Casas Aznar y Bertrán, 2013; Nelson et al., 2007).

A nivel cognitivo y escolar, observan un desarrollo cognitivo más positivo, con niveles de CI mayores, en niños incluidos en familias temporarias que en aquellos que permanecen institucionalizados (Fox et al., 2011; Nelson et al., 2007). Montserrat Boada, Casas Aznar y Bertrán (2013), revelan una menor cantidad de inasistencia a la escuela, menor porcentaje de abandono escolar, menor necesidad de medidas disciplinarias en el aula y mayor posibilidad de encontrarse en el curso correspondiente a la edad cronológica (con menor nivel de repitencia) en adolescentes incluidos en familia temporaria que en adolescentes institucionalizados. En cuanto al desarrollo neurológico y cerebral, un estudio concluye que los niños que ingresaban a familias temporarias mostraban cambios significativos y positivos en su funcionamiento cerebral en áreas cognitivas y socioafectivas (McLaughlin et al., 2011). En el área afectiva y socio relacional se concluye que el cuidado de una familia interviene generando mayores niveles de satisfacción y mirada más positiva ante los cuidados recibidos que en los niños que se mantienen institucionalizados, promoviendo habilidades de comunicación social, aumentando de manera significativa la conexión y vínculos con el otro, disminuyendo riesgos de desarrollar trastornos del espectro autista, depresivos y los niveles de inhibición durante conversaciones sociales (Almas et al., 2014; Kang et al., 2014; Levin et al., 2015).

Otros aspectos investigados en relación a los programas de familias temporarias han sido en torno a las características de las familias temporarias y su relación con el desarrollo del niño. La revisión permite concluir que la familia debería reunir ciertas características para facilitar el acogimiento y consecuencias favorables en el niño, entre ellas se encuentran: aceptación y respeto ante la vinculación del niño con su familia de origen, predominio de deseo de realizar un acto de solidaridad (Herce et al., 2003), posibilidad de mantener estabilidad emocional y construir vínculos seguros entre los miembros de la familia, con el niño y con el mundo exterior, con capacidad de comprender las necesidades del niño y satisfacerlas e integrarlo y generar en él un sentido de pertenencia al grupo familiar, exis- 
tencia de hijos en el núcleo de convivencia, altos niveles de motivación en los adultos de la familia para ser parte del programa (Dumitrescu, 2016; Molero Mañes, Gil Llario y Díaz Rodríguez, 2014; Molero Mañes y Moral Valderas, 2009). También se ha revelado la importancia de disponer de buenas condiciones de vida y de recibir beneficios y aportes de organizaciones o gobierno (Dumitrescu, 2016). En Mendoza, Argentina, una investigación realizada por Valgañón (2014) permite concluir la necesidad de que las familias temporarias presenten un funcionamiento salutógeno con las siguientes características: permeabilidad ante el cambio (sin vivenciarlo como una amenaza), capacidad de conexión con diversos grupos sociales y de fomentar experiencia relacional, habilidad para estimular expresión de las distintas habilidades de sus miembros y ser poseedoras de una organización con liderazgos claros y democráticos.

A modo de cierre del presente apartado sobre el tipo de cuidado y desarrollo del niño enprogramas de familias temporarias, es importante mencionar que, si bien este tipo de programa existe en diversos países del mundo, siendo sostenido por varios fundamentos, el acogimiento residencial o institucional sigue siendo la medida predominante a nivel mundial, quedando muchos desafíos y retos por investigar y gestionar (Anghel, Herczog y Dima, 2013; Del Valle, Bravo y López López, 2009; López López et al., 2010; López López et al., 2011; Palummo, 2013).

\section{Conclusiones}

El presente estudio de revisión bibliográfica, ha intentado acercarnos a la elaboración de una síntesis sobre el desarrollo de niños, niñas y adolescentes bajo cuidados alternativos, tanto institucionalizados como incluidos en programas de acogimiento familiar temporario.

Uno de los aspectos que se desprende de ello, es la existencia de diversas fallas en el cuidado establecido hacia los NNA institu- cionalizados. Se especifica como el carecer de una figura de cuidado exclusiva, estable, significativa, disponible y sensible para el niño, conlleva a retrasos y dificultades en el desarrollo y salud psicofísica. Se ha logrado detallar retrasos y complicaciones en diversas áreas del desarrollo tales como: cognitiva, socioafectiva, física y neurológica.

Otro aspecto que también se destaca es la existencia de programas de familia de acogida temporal en diversos países del mundo, incluyendo distintos continentes. A su vez, en cuanto a la fundamentación de la existencia de los mismos, la lectura bibliográfica de los artículos seleccionados permite realizar un aporte en la presente revisión. Más específicamente, se lograron clasificar los fundamentos en tres categorías. La primera de ella, es una categoría jurídica y legal, desde la cual se impulsa y sostienen los programas de familia temporaria a partir del reconocimiento y protección del derecho del niño a vivir en un ambiente familiar. La segunda, es la categoría correspondiente a una fundamentación preventiva, evitando así los efectos negativos de la institucionalización en el desarrollo de los niños. Por último, la tercera categoría, se refiere a aquellos fundamentos generados por estudios comparativos que permiten concluir cambios positivos del grupo de niños institucionalizados tras su inclusión a familia temporaria, o bien, desarrollo más saludable en niños acogidos en familias temporarias que en instituciones. Con respecto a esto último, se destacan cambios favorables a nivel del desarrollo físico, neurológico, cognitivo, socioemocional y comportamental.

Para finalizar es importante destacar que la presente revisión pretende ser un punto de partida para posteriores investigaciones, tomando en consideración los diferentes hallazgos mencionados. En base a ello, nuevas líneas de estudio podrían considerar los siguientes puntos:

Por un lado, es importante revisar y profundizar sobre estudios longitudinales, que permitan focalizarse en un mismo grupo 
de niños institucionalizados a lo largo del tiempo, para identificar los cambios en su desarrollo tras su posterior ingreso a la familia temporaria. En este aspecto, sólo un estudio fue encontrado con estas características.

A su vez, sería valioso generar estudios con franjas etarias menos amplias (0-3 meses, 4-6 meses, etc.), ya que los parámetros del desarrollo de los niños varían enormemente según la etapa en la que se encuentre.

Asimismo, sería necesario describir los distintos motivos por los cuales se lleva a cabo el ingreso de niños y niñas en cuidados alternativos en los distintos países, tales como: vulneración de derechos, maltrato físico, abuso sexual, negligencia, entre otros. En este sentido, podría también realizarse estudios descriptivos asociativos y correlacionales, con el objeto de establecer relaciones entre la historia del niño o niña previo al ingreso en cuidados alternativos con el desarrollo psicomotor y físico, identificando cómo los diversos motivos que llevaron al ingreso a un hogar o programa de acogimiento familiar temporario, se relacionan con el desarrollo psicomotor durante la estadía en dicho ámbito.

Por último, sería de vital importancia, investigar sobre propuestas de intervención psicosociales en ámbitos de cuidados alternativos institucionales, identificando aquellos que promuevan cambios vinculares entre niños y niñas y personal de cuidadores, posibilitando una contención y cuidado más favorable y salutógeno.

\section{Referencias bibliográficas}

Almas, A.N., Degnan, K.A., Walker, O.L., Radulescu, A, Nelson, C.A., Zeanah, C.H. y Fox, N.A. (2014). The effects of early institutionalization and foster care intervention on children's social behaviors at the age of eight. Social Development, 24(2), 225-239. doi: 10.1111/sode. 12089

Amorós, P., Palacios, J., Fuentes, N., León, E. y Mesas, A. (2003). Familias canguro: Una experiencia de protección a la infancia. Barce- lona: Fundación La Caixa.

Anghel, R., Herczog, M. y Dima, G. (2013). The challenge of reforming child protection in Eastern Europe: The cases of Hungary and Romania. Psychosocial Intervention, 22(3), 239-249. doi: 10.5093/in2013a27

Bakermans-Kranenburg, M.J., Steele, H., Zeanah, C.H., Muhamedrahimov, R.J., Vorria, P., Dobrova-Krol N.A., Steele, M., Van IJzendoorn, M.H., Juffer, F. y Gunnar, M.R. (2011). Attachment and emotional development in institutional care: Characteristics and catch-up. Monographs of the Society for Research in Child Development, 76(4), 62-91. doi: 10.1111/j.1540-5834.2011.00628.x

Beckett, C., Bredenkamp, D., Castle, J., Groothues, C., O'Connor, T.G. y Rutter, M. (2002). Behavior patterns associated with institutional deprivation: a study of children adopted from Romania. Journal of Developmental and Behavioral Pediatrics, 23(5), 297-303. doi: 10.1097/00004703-200210000-00001

Browne, E. (2017). Children in care institutions. K4D Helpdesk Report. Brighton, UK: Institute of Development Studies. Recuperado de https://opendocs.ids.ac.uk/opendocs/ handle/123456789/13208

Cartoceti, R., Abusamra, V., De Beni, R. y Cornoldi, C. (2016). Comprensión de textos en contextos desfavorecidos: El efecto de un programa de intervención en la habilidad para detectar errores e incongruencias en textos escritos. Interdisciplinaria, 33(1), 111-128. Recuperado de http://www. scielo.org.ar/scielo.php?script=sci_arttext\&pid=S1668-70272016000100007. doi: 10.16888/interd.2016.33.1.7

Chugani, H.T., Behen, M.E., Muzik, O., Juhász, C., Nagy, F. y Chugani, D.C. (2001). Local brain functional activity following early deprivation: A study of postinstitutionalized Romanian orphans. Neuroimage, 14(6), 1290-1301. doi: 10.1006/nimg.2001.0917

Del Valle, J.F., López López, M., Montserrat, C. y Bravo, A. (2008). El acogimiento familiar en España. Una evaluación de resultados. Madrid: Ministerio de Trabajo y Asuntos Sociales 
Del Valle, J.F., Bravo, A. y López López, M. (2009). El acogimiento familiar en España: Implantación y retos actuales. Papeles del Psicólogo, 30(1), 33-41. Recuperado de http:// www.redalyc.org/articulo.oa?id=77811388005

Dumitrescu, A.M. (2016). The life quality of a child from a foster family in Romania. International E-Journal of Advances in Social Sciences, 2(4), 204-211. doi: 10.18769/ijasos

Eluvathingal T.J., Chugani H.T., Behen, M.E., Juhász, C., Muzik, O., Maqbool, M., Chugani, D.C. y Makki, M. (2006). Abnormal brain connectivity in children after early severe socioemotional deprivation: a diffusion tensor imaging study. Pediatrics, 117(6), 2093-2100. doi: 10.1542/peds.20051727

Fernández Millán, J.M., Hamido-Mohamed, A. y Ortiz Gómez, M.M. (2009). Influencia del acogimiento residencial en los menores en desamparo. Electronic Journal of Research in Educational Psychology, 7(18), 715-728. Recuperado de http:/www.redalyc.org/ html/2931/293121945006/

Finet, C., Vermeer, H.J., Juffer, F., Bosmans, G. y Bijttebier, P. (2016). Children's cognitive development after adoption. En F. Genesse and A. Delcenserie (Ed.), Starting Over The Language Development in Internationally-Adopted Children (pp. 37-62). Montreal, Canada: Trends in Language Acquisition Research.

Fox, N.A., Almas, A.N., Degnan, K.A., Nelson, C.A. y Zeanah, C.H. (2011). The effects of severe psychosocial deprivation and foster care intervention on cognitive development at 8 years of age: findings from the Bucharest Early Intervention Project. Journal of the American Academy of Child and Adolescent Psychiatry, 52(9), 919-928. doi: 10.1111/j.14697610.2010.02355.x

García-Baamonde Sánchez, M.E. (2008). Análisis de la competencia lingüística y de la adaptación personal, social, escolar y familiar en niños institucionalizados en centros de acogida. (Tesis doctoral). Universidad de Extremadura, España.
García Quiroga, M. y Hamilton-Giachritsis, C. (2016). Attachment styles in children living in alternative care: A systematic review of the literature. Child \& Youth Care Forum, 45(4), 625-653. Recuperado de https://link.springer. com/article/10.1007/s10566-015-9342-x

Gómez Bengoechea, B. y Berástegui Pedro Viejo, A. (2009). El derecho del niño a vivir en familia. Miscelánea comillas: Revista de Ciencias Humanas y Sociales, 67(130), 175-198. Recuperado de http://revistas.upcomillas.es/ index.php/miscelaneacomillas/article/view/894

Govindan R.M., Behen M.E., Helder E., Makki M.I. y Chugani H.T. (2010). Altered water diffusivity in cortical association tracts in children with early deprivation identified with Tract-Based Spatial Statistics (TBSS). Cerebral Cortex, 20(3), 561-569. doi: 10.1093/ cercor/bhp122

Groark, C.J., McCall, R.B., Fish, L. y Whole Child International Evaluation Team. (2011). Characteristics of environments, caregivers, and children in three central American orphanages. Infant Mental Health Journal, 32(2), 232-250. doi: 10.1002/imhj.20292

Gunnar, M. y Quevedo, K. (2007). The neurobiology of stress and development. Annual Review of Psychology, 58, 145-173. doi: 10.1146/ annurev.psych.58.110405.085605

Heim, C. y Nemeroff, C.B. (2001). The role of childhood trauma in the neurobiology of mood and anxiety disorders: preclinical and clinical studies. Biological Psychiatry, 49(12), 10231039. doi: 10.1016/S0006-3223(01)01157-X

Herce, C., Achucarro, C., Gorostiaga, A., Torres Gómez de Cádiz, B. y Balluerka, N. (2003). La integración del menor en la familia de acogida: factores facilitadores. Psychosocial Intervention, 12(2), 163-177. Recuperado de http://www.redalyc.org/articulo. oa?id=179818034004

Jaar, H.E. y Córdova, V.M. (2017). Prevención de la carencia afectiva crónica: Nuevos paradigmas en el modelo de familia de acogida temporal. Revista Chilena de Neuropsiquiatría, 55(1), 44-51. doi: 10.4067/S071792272017000100006 
Johnson, D.E. y Gunnar, M.R. (2011). IV. Growth failure in institutionalized children. Monographs of the Society for Research in Child Development, 76(4), 92-126. doi: 10.1111/j.1540-5834.2011.00629.x

Kang, H., Chung, I.J., Ichun, J.S., Choong, R. y Woo, S. (2014). The outcomes of foster care in South Korea ten years after its foundation: A comparison with institutional care. Children and Youth Services Review, 39, 135-143. doi: 10.1016/j.childyouth.2014.01.024

Kaufman, J., Plotsky, P.M., Nemeroff, C.B. y Charney, D.S. (2000). Effects of early adverse experiences on brain structure and function: clinical implications. Biological Psychiatry, 48(8), 778-790. doi: 10.1016/S00063223(00)00998-7

Kertes, D.A., Gunnar, M.R., Madsen, N.J. y Long, J.D. (2008). Early deprivation and home basal cortisol levels: a study of internationally adopted children. Development and Psychopathology, 20(2), 473-491. doi: 10.1017/ S0954579408000230.

Levin, A.R., Fox, N.A., Zeanah, C.H. y Nelson, C.A. (2015). Social communication difficulties and autism in previously institutionalized children. Journal of the American Academy of Child and Adolescent Psychiatry, 54(2), 108-115. doi: 10.1016/j.jaac.2014.11.011.

Ley $N^{\circ}$ 6.354. Ley de Protección integral del niño y del adolescente. Boletín Oficial del Gobierno de Mendoza, 28 de diciembre del 1995.

Ley $\mathrm{N}^{\circ} 26.061$. Ley de protección integral de los derechos de las niñas, niños y adolescentes. Buenos Aires, Argentina, 21 de octubre del 2005.

Ley $\mathrm{N}^{\circ}$ 2.213. Ley de Acogimiento Familiar. Boletín Oficial del Gobierno de la Ciudad de Buenos Aires, 26 de enero del 2007.

López López, M., Montserrat Boada, C., Del Valle, J. y Bravo, A. (2010). El acogimiento en familia ajena en España. Una evaluación de la práctica y sus resultados. Infancia y Aprendizaje, 33(2), 269-280. doi: 10.1174/021037010791114616

López López, M., Del Valle, F.J., Montserrat Boada, C. y Bravo Arteaga, A. (2011). Niños que esperan. Estudio sobre casos de larga estancia en acogimiento residencial. Madrid: Minis- terio de Sanidad y Política Social. Recuperado de http://www.observatoriodelainfancia.es/oia/ esp/documentos_ficha.aspx?id=3409

Márquez, M.S., Iparraguirre, M.S. y Bengtsson, A.M. (2015). Un recorrido por el aprendizaje de la escritura desde una perspectiva evolutivo-educativa y semiótica. Interdisciplinaria, 32(1), 151-168. Recuperado de http://www. scielo.org.ar/scielo.php?script=sci_arttext\&pi$\mathrm{d}=\mathrm{S} 1668-70272015000100008 \& \ln \mathrm{g}=$ es\&tln$\mathrm{g}=\mathrm{es}$

Martín, E., Muñoz de Bustillo, M.C., Rodríguez, T. y Pérez, Y. (2008). De la residencia a la escuela: la integración social de los menores en acogimiento residencial con el grupo de iguales en el contexto escolar. Psicothema, 20(3), 376-382. Recuperado de http://www. redalyc.org/html/727/72720306/

Martín, E., Muñoz de Bustillo Díaz, M.C. y Pérez, N. (2011). Las relaciones de amistad en la escuela de los menores en acogimiento residencial. Revista de Psicodidáctica, 16(2), 351-366. Recuperado de https://dialnet. unirioja.es/servlet/articulo? codigo $=3674151$

Martín, E., García, M.D. y Siverio, M.A. (2012). Inadaptación autopercibida de los menores en acogimiento residencia. Anales de Psicología, 28(2), 541-547. doi: 10.6018/ analesps.28.2.148921

Martínez González, C. (2008). Acogimiento familiar, ¿justicia o solidaridad? Pediatría Atención Primaria, 10(40), 129-136. Recuperado de https://dialnet.unirioja.es/servlet/articulo?codigo $=2946192$

McCall, R.B. (2012). The development and care of institutionally reared children. The Leiden conference on the development and care of children without permanent parents. Child Development Perspectives, 6(2), 174-180. doi: 10.1111/j.1750-8606.2011.00231.x

McLaughlin, K.A., Fox, N.A., Zeanah, C.H. y Nelson, C.A. (2011). Adverse rearing environments and neural development in children: The development of frontal electroencephalogram asymmetry. Biological Psychiatry, 70(11), 1008-1015. doi: 10.1016/j. biopsych.2011.08.006 
Mehta, M.A., Golembo, N.I., Nosarti, C., Colvert, E., Mota, A., Williams, S.C., Rutter, M. y Sonuga-Barke, E.J. (2009). Amygdala, hippocampal and corpus callosum size following severe early institutional deprivation: the English and Romanian adoptees study pilot. Journal of Child Psychology and Psychiatry, 50(8), 943-951. doi: 10.1111/j.1469-7610.2009.02084.x.

Molero Mañes, R.J. y Moral Valderas, M.J. (2009). Factores de protección encontrados en un estudio longitudinal con menores acogidos en familias educadoras. International Journal of Developmental and Educational Psychology, 2(1), 419-425. Recuperado de http://www. redalyc.org/articulo.oa?id=349832321046

Molero Mañes, R.J., Gil Llario, M.D. y Díaz Rodríguez, I.M. (2014). Indicadores de la calidad del proceso de acogimiento familiar. International Journal of Developmental and Educational Psychology, 2(1), 475-481. doi: 10.17060/ijodaep.2014.n1.v2.463.

Monaghan, P. y Haussmann, M.F. (2015). The positive and negative consequences of stressors during early lige. Early Human Development, 91(11), 643-647. doi: 10.1016/j. earlhumdev.2015.08.008.

Montserrat Boada, C., Casas Aznar, F. y Bertran, I. (2013). Desigualdad de oportunidades educativas entre los adolescentes en acogimiento residencial y familiar. Infancia y Aprendizaje, 36(4), 443-454. doi: 10.1174/021037013808200267

Moreno Manso, J.M., Rabazzo Méndez, M.J. y García-Baamonde M.E. (2006). Competencia lingüística y estilo cognitivo en niños institucionalizados. Revista de Logopedia, Foniatría y Audiología, 26(2), 115-125. doi: 10.1016/ S0214-4603(06)70109-7

Moreno Manso, J.M., García-Baamonde Sánchez, M.E. y Blázquez Alonso, M. (2010). Desarrollo lingüístico y adaptación escolar en niños en acogimiento residencial. Anales de Psicología, 26(1), 189-196. Recuperado de http:// www.redalyc.org/html/167/16713758022/

Moulson, M.C., Shutts, K., Fox, N.A., Zeanah, C.H., Spelke, E.S. y Nelson, C.A. (2014). Effects of early institutionalization on the development of emotion processing: A case for relative sparing? Developmental Science, 18(2), 298-313. doi: 10.1111/desc. 12217

Nemeroff, C.B. (2004). Neurobiological consequences of childhood trauma. Journal of clinical psychiatry, 65(1), 18-28. Recuperado de https://www.ncbi.nlm.nih.gov/ pubmed/14728093

Nelson, C.A., Zeanah, C.H., Fox, N.A., Marshall, P.J., Smyke, A.T. y Guthrie, D. (2007). Cognitive recovery in socially deprived young children: the Bucharest early intervention project. Science, 21(318), 1937-1940. doi: 10.1126/ science. 1143921

O’Connor, T.G., Rutter, M., Beckett, C., Keaveney, L. y Kreppner, J.M. (2000). The effects of global severe privation on cognitive competence: extension and longitudinal follow-up. Child development, 71(2), 376-390. Recuperado de https://www.ncbi.nlm.nih.gov/ pubmed/10834471\#

Palacios, J., Moreno, C. y Román, M. (2013). Social competence in internationally adopted and institutionalized children. Early Childhood Research Quarterly, 28(2), 357-365. doi: 10.1016/j.ecresq.2012.08.003

Palummo, J. (2013). La situación de niños, niñas y adolescentes en las instituciones de protección y cuidado de América Latina y el Caribe. Fondo de las Naciones Unidas para la Infancia (UNICEF), Oficina Regional para América Latina y el Caribe, Panamá.

RELAF (2016). Los Olvidados: Niños y niñas en "hogares". Macroinstituciones en América Latina y el Caribe. Recuperado de http://www. relaf.org/material.html

Rodríguez Juárez, G. (2016). Situación de los niños, niñas y adolescentes privados de cuidados parentales en México. Entretextos, $8(22), 5316$. Recuperado de http://entretextos. leon.uia.mx/

Roy, P. y Rutter, M. (2005). Institutional care: associations between inattention and early reading performance. Journal of Child Psychology and Psychiatry, 47(5), 480-487. doi: 10.1111/j.1469-7610.2005.01526.x

Smyke, A.T., Koga, S.F., Johnson, D.E., Fox, N.A., Marshall, P.J., Nelson, C.A. y Zeanah, 
C.H. (2007). The caregiving context in institution-reared and family-reared infants and toddlers in Romania. Journal of Developmental and Behavioral Pediatrics, 48(2), 210-218. doi: 10.1111/j.1469-7610.2006.01694.x

UNICEF. Ministerio de Desarrollo Social y Secretaría Nacional de Niñez, Adolescencia y Familia (2012). Situación de niños, niñas $y$ adolescentes sin cuidados parentales en la República Argentina. Relevamiento nacional y propuestas para la promoción y el fortalecimiento del derecho a la convivencia familiar y comunitaria. Buenos Aires, Argentina.

UNICEF, Ministerio de Desarrollo Social y Secretaría Nacional de Niñez, Adolescencia y Familia (2014). Situación de niños, niñas $y$ adolescentes sin cuidados parentales en la República Argentina. Relevamiento nacionalactualización 2014. Buenos Aires, Argentina.

Valgañón, M. (2014). Estilo de funcionamiento de las familias de acogida y conducta adaptativa. Autoconcepto de los niños, niñas y adolescentes bajo su cuidado. Salud y Sociedad,
5(2), 156-169. Recuperado de https://dialnet. unirioja.es $/$ servlet $/$ articulo? codigo $=4945411$

Van IJzendoorn, M.H., Palacios, J., Sonuga-Barke, E.J.S., Gunnar, M.R., Vorria, P, McCall, R.B., LeMare, L., Bakermans-Kranenburg, M.J., Dobrova-Krol, N. y Juffer, F. (2011). Children in institutional care: Delayed development and resilience. Monographs of the Society for research in child development, 76(4), 8-30. doi: 10.1111/j.1540-5834.2011.00626.x

Velázquez, M.E. (2014). Un recorrido bibliográfico por los conceptos sujeto-sociedad, cuerpo indumentaria y sus interrelaciones. Interdisciplinaria, 31(2), 227-237. Recuperado de http:// www.scielo.org.ar/scielo.php?script $=$ sci arttext\&pid=S1668-70272014000200003. doi: 10.16888/interd.2014.31.2.3

World Health Organization, Department of Child and Adolescent Health and Development (2004). The importance of caregiver-child interactions for the survival and healthy development of young children: a review. Geneva: World Health Organization.

Recibido: 9 de enero de 2018 Aceptado: 15 de octubre de 2019 
